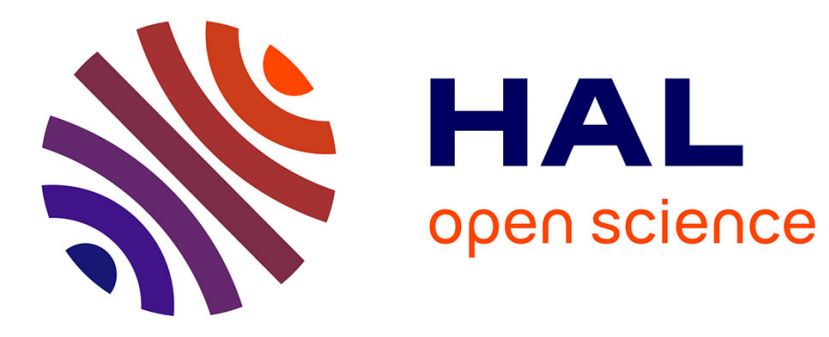

\title{
Green Growth in NDN: Deployment of Content Stores
}

Elian Aubry, Thomas Silverston, Isabelle Chrisment

\section{To cite this version:}

Elian Aubry, Thomas Silverston, Isabelle Chrisment. Green Growth in NDN: Deployment of Content Stores. LANMAN 2016 - IEEE International Symposium on Local and Metropolitan Area Networks, IEEE, Jun 2016, Rome, Italy. 10.1109/LANMAN.2016.7548850 · hal-01405820

\section{HAL Id: hal-01405820 \\ https://hal.science/hal-01405820}

Submitted on 6 Dec 2016

HAL is a multi-disciplinary open access archive for the deposit and dissemination of scientific research documents, whether they are published or not. The documents may come from teaching and research institutions in France or abroad, or from public or private research centers.
L'archive ouverte pluridisciplinaire HAL, est destinée au dépôt et à la diffusion de documents scientifiques de niveau recherche, publiés ou non, émanant des établissements d'enseignement et de recherche français ou étrangers, des laboratoires publics ou privés. 


\title{
Green Growth in NDN: Deployment of Content Stores
}

\author{
Elian Aubry ${ }^{\dagger} *$, Thomas Silverston ${ }^{\ddagger}$ and Isabelle Chrisment ${ }^{\dagger} *$ \\ †Université de Lorraine, LORIA - CNRS UMR 7503, Vandœuvre-lès-Nancy, France \\ * Inria - Nancy Grand-Est, Villers-lés-Nancy, F-54600, France \\ ¥The University of Tokyo, JFLI - CNRS UMI 3527, Tokyo, Japan
}

\begin{abstract}
Named-Data Networking architecture relies on cache networks, where nodes store the data for further requests. However, the memory needed at each node called Content Store represents the most significant part of the entire cost of the infrastructure that has to be supported by network providers, making difficult the change from the current Internet infrastructure to a Future Internet based on NDN. Thus, a legitimate question would be: "are all these Content Stores useful in a large-scale NDN network?" In this paper, we investigate the impact of Content Stores in NDN network, and we evaluate the performances of the NDN architecture according to the number of Content Stores effectively deployed in the network. We show through extensive simulation experiments in NS-3 that only about $50 \%$ of nodes with Content Stores is enough to achieve higher level of performances than a fully-deployed NDN network. This result is very important for the deployment of NDN architecture as it shows that the infrastructure cost can be drastically reduced and it is an incentive for network providers that benefits directly from this result.
\end{abstract}

\section{INTRODUCTION}

Named-Data Networking architecture (NDN) [[1], [2]] has its roots in an earlier project called Content-Centric Networking $(\mathrm{CCN})$, and has been recently proposed as an alternative to the classical TCP/IP host-to-host communication model for a Future Internet. NDN focuses on the content itself and not the location of hosts; packets address names and not hosts and content is stored at each node along the delivery path (innetwork caching); any node in the network can therefore issue data for further requests. Besides Interest and Data messages, each NDN node has three components: a cache memory called Content Store (CS), a table to store forwarding information Forwarding Interest Base (FIB), and a table to deliver the content up to the user Pending Interest Table (PIT).

As NDN is a cache networking architecture, Content Stores count therefore for one of the main part of the infrastructure cost, and this is an issue for network operators to deploy the NDN architecture at Internet scale. In addition, cache memory at each node generates high energy demand, and leads to important infrastructure and operational costs for network providers (CAPEX/OPEX).

Numerous studies have focused on caching in NDN, such as caching strategies or cache allocation problems ( [3], [4], [5], [6], [7], [8], [9], [10], [11]). These studies aim to help the decision at each nodes whether to cache content or not. However, to the best of our knowledge, there has been no prior work to address the efficiency of NDN network with regards to overall cache capabilities in the network.

In this paper, we evaluate the performances of NDN architecture according to the number of Content Stores in the network. The rationale of this research work is to answer legitimate questions such as "how many Content Stores (i.e., nodes with caching capabilities) allows the best efficiency in a large-scale NDN network?" To this end, we conducted extensive simulation experiments with NS-3 simulator, using ISP-like topologies, with several scenarios and by varying the number of Content Stores deployed in the network. Our results show that only a subset of nodes (e.g.: 50\%) is sufficient enough to achieve highest caching performances; therefore there is no need to have all the nodes equipped with caching capabilities. This result is an important finding for network operators to deploy the NDN infrastructure, as it shows that hardware equipment cost (e..: cache memory) can be drastically reduced.

The reminder of the paper is organized as follows. Section II presents the related work and Section III provides an overview of the NDN architecture. We describe our experiments in Section IV and the results in Section V. Section VI discusses our finding and next step of this research work, while Section VII concludes the paper.

\section{RELATED WORK}

Information Centric Networking has recently attracted a lot of interest in the research community and especially the NDN architecture [2]. As this new network paradigm relies mostly on the use of cache in the network, there has been a lot of studies focusing on the design of novel caching strategies, or cache allocation.

For example, Chai et al. [3] show that by randomly caching in a single node along the delivery path and "caching less", they achieve similar level of performances while consuming less resources (e.g.: memory). In order to improve the cache performances in NDN, Bernardini et al. [4] propose MPC, a strategy caching only popular content, while SACS [5] aims at caching content produced by popular users. Ren et al. [6] propose MAGIC, a strategy enabling to reduce the number of caching operation and the bandwidth consumption. Rezazad et al. [7] present CCndnS, a caching strategy for CCN spreading content to increase diversity in Content Stores. All these works aim at improving the simple default caching strategy in NDN 
architecture, where all nodes keep a local copy of the content that has traveled through (Leave Copy Down).

Regarding cache allocation, Rezazad et al. [9] propose to separate the NDN Content Store into different classes and allocate the node's memory accordingly. Fayazbakhsh et al. [12] evaluate the gain provide by several factors in ICNs like cache placement (edge-cache VS pervasive caching) or routing (shortest path to origin servers VS nearest replica). They proved that pervasive and edge-based cache give similar performances, and propose a solution to deploy ICN incrementally without changing the core-network. In other hand, Wang et al. [13] investigate the optimal cache allocation problem for $\mathrm{CCN}$ and give an algorithm to distribute cache capacity across the network. They also evaluate impact of topology, network size, content popularity and object replacement strategies. Differently from [12], they find that cache capacity must be placed on central nodes of the network. According to Wang et al., an homogeneous cache-size at each nodes cannot be optimal and depends directly on the topology.

Rossini et al. [11] also compare homogeneous and heterogeneous cache size in $\mathrm{CCN}$ and show that the cache size allocation depends on the graph centrality metrics such as closeness, stress and the centrality degree.

All these previous studies assume that all nodes do have caching capabilities, i.e. a Content Store, and proposed new mechanisms that will help the caching decision at each node and improve the overall NDN performances.

Regarding NDN deployment, Perino et al. [14] make an evaluation of the suitability of existing software and hardware components in routers for the support of CCN. They show that supporting an Internet-scale $\mathrm{CCN}$ deployment is not easily feasible, whereas a Content Delivery Network (CDN) at ISPscale can be easily afforded. Jiang et al. [15] propose to use NDN architecture to enhance CDN. Their solution nCDN runs over TCP/UDP protocols and show its efficiency to overcome traditional CDN framework with regards to scalability, reliability and quality of services. Wu et al. [16] integrate NDN forwarding scheme within IP network to reduce energy consumption.

Differently, in our work, we do not only consider each node and its caching capabilities but we address the overall cache network and its performances. Our following experiments will show that there is no need all nodes have caching capabilities to achieve high level of performances. As memory is an important part of the NDN infrastructure cost, this shows that NDN architecture can be deployed at reduced cost.

\section{NDN OVERVIEW}

The Internet is now mostly used to access content [17] and it was originally designed based on a host-to-host paradigm. Then, the Named-Data Networking architecture (NDN) [2] has recently been proposed to have the content at the core of the communication, and is based on a hostto-content paradigm. Packet messages address content names and not host as with the current Internet Protocol. For example, Content names are described as URL as follows:

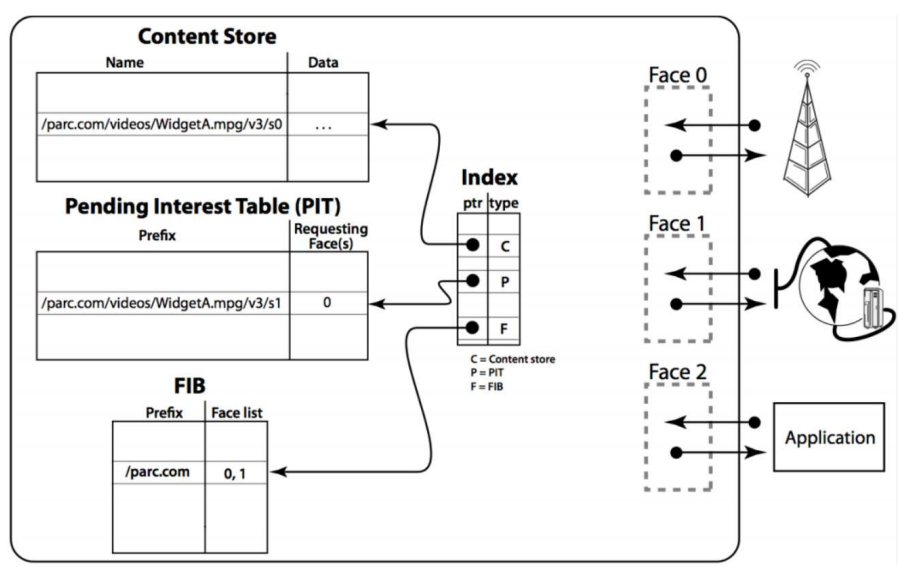

Fig. 1: Architecture of a NDN node as proposed in [1]

\section{/youtube/example/video/cat.avi}

The NDN architecture relies mainly on two messages, Interest and Data. When a user wants to retrieve a content, she sends an Interest into the network, which will be forwarded to a node possessing the content in its Content Store. Then, Data are sent back on the reverse path to the requester.

NDN nodes are composed of three components as proposed by Jacobson et al. [1] and shown on Figure 1: the Content Store (CS), a cache memory that stores local copy of content; the Forwarding Information Base (FIB) that contains entries to forward the requests (.i.e: Interest). Each entry is composed of a prefix and all the network interfaces (the faces) where this content can be found; and the Pending Interest Table (PIT), a table similar to the FIB that keeps information on the faces where to transmit the Data messages back to the users.

Thus, when a node receives an Interest, if it has the content described by the prefix in its cache (CS), it returns a Data message to the user through the same interface. Otherwise, the node looks into its FIB to find an entry that matches with the content name and forwards the Interest into the network. If there is no entry, the Interest message is forwarded on all its interfaces (i.e: broadcasted). Note that forwarding in NDN is still an open issue; there has been several proposals [18], [19] but this topic is out of the scope of this paper. While receiving an Interest message, the nodes update their PIT to be able to forward the Data on the reverse path up to the requester.

Finally, all the nodes receiving a Data message can store the content into their Content Store, according to a caching strategy. The default NDN caching strategy enables nodes to store all the content that has traveled through (Leave Copy Down). If the Content Store is full, a cache policy is used to drop a content from memory (e.g.: Least-Frequently Used, Last-Recently Used, etc.).

\section{EXPERIMENTS}

In this section, we present our experiments conducted to evaluate the impact of the number of nodes with caching capabilities (i.e. Content Stores) in the network. The rationale of this work is that with only a subset of nodes being able to store content, the performances of the NDN network are 


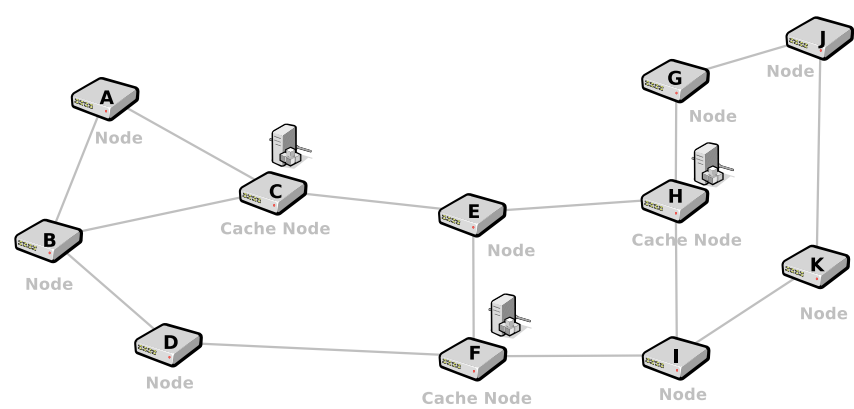

Fig. 2: Example of considered NDN network: topology is an ISPlike network (ex: Abilene) and only nodes $\mathrm{C}, \mathrm{F}, \mathrm{H}$ have caching capabilities (i.e.: Content Stores).

still high. Therefore, there is no need to deploy Content Stores at each node, reducing de-facto the entire cost of the NDN network infrastructure, as memory is the main cost for new hardware. Indeed, as it is the case in economy and environmental sciences, Green Growth [20] can allow achieving the same level of development - or performances in our case, by more "efficient use of natural resources" (i.e.: without wasting resources).

Our considered case is described on Figure 2. In this example, an ISP-like network is composed of NDN nodes, and only some of them have caching capabilities (i.e.: nodes $\mathrm{C}, \mathrm{F}$ and $\mathrm{H})$. Other NDN nodes do not have Content Stores, and can just forward Interest and Data messages. In the rest of the paper, will refer to regular NDN nodes possessing a Content Store as "Cache Nodes" (i.e.: nodes C,F,H); we will refer to NDN node without a Content Store as "Node" (i.e.: nodes A,B,D,E,G,I,J,K).

We have considered scenarios where the number of cache nodes (i.e.: nodes with a Content Store) varies from $0 \%$ to $100 \%(0 \%, 20 \%, 40 \%, 50 \%, 80 \%$ and $100 \%)$ and cache nodes are randomly distributed into the network topology. In the "0\%" scenario, there is no Content Store in the network, then no nodes have caching capability. Thus, all the Interest messages should therefore reach the origin server to obtain a content. This scenario reminds the traditional IP network scenario where a request from an host should reach a target server. This scenario is tested as a boundary, as cache network such as NDN will necessarily count Content Stores in its infrastructure. Differently, in the $100 \%$ scenario, all cache nodes have Content Stores, which remind the regular NDN behavior where all nodes cache content. In the other scenarios, only $20 \%, 40 \%, 50 \%$ or $80 \%$ of nodes have Content Store. In addition, we also have considered scenarios where only a single node of the network has a Content Store, and we distinguish two cases: the case where the node with Content Store is randomly placed on the network (Scenario "1"); and the case where the most-connected node in the network has the Content Store (Scenario "1MC"). These scenarios remind the Content Distribution Network (CDN) scenario in IP network with additional CDN server deployed to help origin server distributing content.
Then we evaluate the performances of the NDN network under all these scenarios by using the traditional metrics such as the Cache Hit Ratio (Equation 1), the total number of messages in the network, and the delay to reach a content. For each node of the network, called $n_{i}$, we note $I_{i}$ the number of requests (i.e. Interest message) received by $n_{i}$ and $D_{i}$ the number of responses (i.e. Data message) returned by the node $n_{i}$ 's Content Store. The Cache Hit Ratio $(\mathrm{CHR})$ represents the global caching system of the network (all the nodes) and can be calculates by the sum of all Responses $D_{i}$ divided by the sum of all Interest $I_{i}$ :

$$
C H R=\frac{\sum D_{i}}{\sum I_{i}}
$$

Cache Hit ratio allows evaluating the performances of the cache network and the probability to find content in cache nodes; the number of messages (Interest and Data) allow evaluating the communication cost (overhead) in the network and if the network resources are wasted with additional messages; Then the delay allow evaluating the time it takes to retrieve a content, e.g., if it is possible to get content closer.

\begin{tabular}{|c|c|}
\hline Parameters & Values \\
\hline Topologies & ISP (Abilene, Geant) \\
\hline Catalog Size & $10^{6}$ \\
\hline Number of Catalog & 1 \\
\hline Number of Users & $10^{3}$ \\
\hline Cache Size & MZipf $(\alpha=1.1, \beta=0)$ \\
\hline Popularity Model & 5 per sec. \\
\hline Request Rate & LCD \\
\hline Caching Strategy & LFU \\
\hline Replacement Policy & $1,1 \mathrm{MC}, 0 \%, 20 \%, 50 \%, 80 \%, 100 \%$ \\
\hline Scenarios & Cache Hit, \#Messages, Delay \\
\hline Metrics & 20 \\
\hline Simulation runs &
\end{tabular}

TABLE I: Simulation Parameters

In our experiments, we use NS-3 [21], a discrete-event network simulator and we run simulations using the ndnSIM module provided by NS-3, enabling to simulate the NDN architecture. We have derived our simulation parameters by using values frequently used in NDN research work [22], [23], [11].

The network topologyis based on both Geant (41 nodes) and Abilene (11 nodes) topologies. Our catalogue of content counts 1 million elements, and the size of Content Store (cache size) represents $0.1 \%$ of the catalog (i.e.: 1,000 elements), a commonly used ratio between catalog and cache size.

We used the default caching strategy in NDN, Leave Copy Down, where each cache node stores content that has traveled through, while the replacement policies is LFU (LeastFrequently Used). In our simulations, 40 users send Interests for content and their request rate is 5 per second. The popularity of the catalog follows a MZipf distribution with $\alpha=1.1$, which is a commonly observed value.

For all experiments, we performe 20 simulation runs per scenario, where users, Content Stores and one Catalog are randomly placed on the network, and we provide average 
values and confidence intervals. All our simulation parameters are summarized in table I.

\section{Simulation Results}

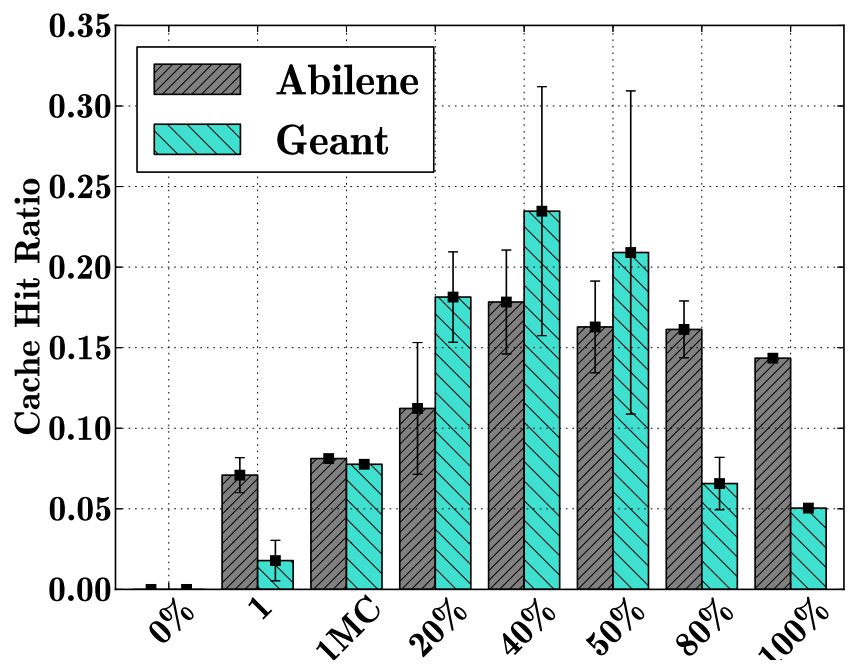

Fig. 3: Cache Hit Ratio

We detail below the results of our experiments. We analyze the impact of the number of Content Stores on the network performances with regards to the metrics Cache Hit Ratio, number of messages (Interests and Data) and the Delay to reach a content.

Cache Hit Ratio: Figure 3 depicts the Cache Hit Ratio when the percent of Cache Nodes varies from "0\%" to "100\%", for Abilene topology (grey bar) and Geant topology (blue bar).

In the Scenario "0\%", The Cache Hit Ratio is null as expected since there is no Content Store in the networks. With a single Content Store in the network (Scenario "1" and "1MC"), results differs with the two different topologies. With Abilene, Scenario "1" and Scenario "1MC" exhibit similar Cache Hit Ratio (0.07), while results are different with Geant and Scenario "1MC" reaches a higher Cache Hit. This is due to the fact that Geant is a more centralized topology and the mostconnected node in this topology has much more connections with other nodes; then it receives more Interest messages and stores more content for further requests, increasing the Cache Hit Ratio of the overall network.

Then, increasing the number of Content Stores in the network with Scenario "20\%" or "40\%" increases the Cache Hit Ratio for both topologies: up to 0.18 for Abilene and 0.24 with Geant. Besides "40\%" of Content Stores in the network, the Cache Hit Ratio decreases down to 0.14 for Abilene and 0.05 for Geant (Scenarios "50\%", "80\%" and "100\%").

This can be explained as follows. Upon receiving an Interest, each cache node (i.e.: with Content Stores) possessing the requested content responds with Data back on the reverse path; and Data messages update the FIB of nodes on the path for further requests. However, when another Interest for the same content arises, cache nodes may have evicted this content from their Content Store while other nodes' FIB stay inconsistent, forwarding the Interest to wrong cache nodes, increasing the number of miss in the network, i.e., reducing the Cache Hit Ratio in the network. This phenomenon is more accentuated with Geant topology, as Cache Hit Ratio decreases drastically from Scenario "50\%" to "80\%", because the topology is highly centralized and counts a larger number of links than Abilene.

This phenomenon has also been observed in another study [24], as caching redundancy has a negative impact on Cache Hit. Indeed, Content Stores tend to store only popular content, all of them having similar elements in memory. Thus, requests to unpopular content are not satisfied, reducing defacto the Cache Hit in the network. This result shows that increasing the number of Content Store in the network does not necessarily improve the performances of the network, but even worse, can reduce the usefulness of caching in the network.

Number of Messages: We investigate the number of messages sent throughout the network (Interest and Data) according to each scenario. Figure $4 \mathrm{a}$ and Figure $4 \mathrm{~b}$ represent the number of Interest and Data messages sent by all the nodes in Abilene, and, Figure 5a and Figure 5b in Geant topology.

Regarding the number of Interest messages, it decreases when the number of Content Store increases. With Abilene, the number of Interest messages are divided by two from Scenario "0\%" to Scenario "20\%". Besides $20 \%$ of cache nodes, the number of Interest continues decreasing but more slowly. Indeed, when there is no Content Store (Scenario "0\%"), Interests are flooded in the Network, and multiple copies of the same Data can be returned to the users, wasting network link's resources. By increasing the number of Content Stores, more content will be stored at different locations and reachable, reducing the number of multiple transmissions of Data and forwarded Interests. In our experiment, $40 \%$ of Content Stores is a threshold and we observe no significant improvement beside this value. Popular content frequently requested are already present in the Content Stores, and more Content Stores will not improve significantly the performances of the network. For Geant, the number of Interest messages also decreases with the number of Content Stores. With "50\%" of Content Stores, the number of Interests has been divided by 6 , and it keeps decreasing up to a threshold of "80\%". As Geant counts much more nodes and links, increasing the storing capabilities have a more sharp impact on the network performances and the reduction of messages. But as we have seen with the Cache Hit Ratio, with more than $50 \%$ of Cache Nodes, cache efficiency is greatly diminished.

The number of Data messages follows the same behaviour than Interest messages and decreases while the number Content Stores increases. For Geant, there is a bump with scenario "20\%" because by adding more Content Stores, more Interests will be resolved by several Data messages from different cache nodes, increasing the number of Data messages with regards to scenarios "0\%", "1" and "1MC".

Delay: Last, we investigate the delay to reach a content, represented on Fig 4c for Abilene and Fig 5c for Geant. Our Delay metric represents the average of all delay from an 


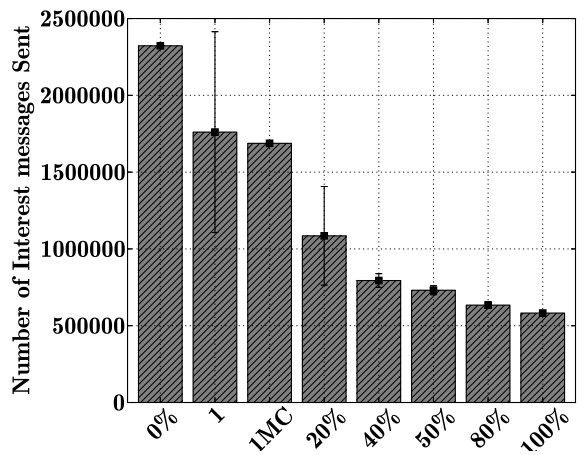

(a) Messages Interest

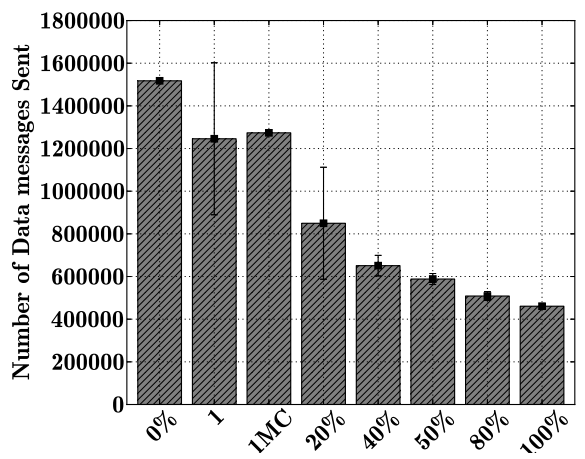

(b) Messages Data

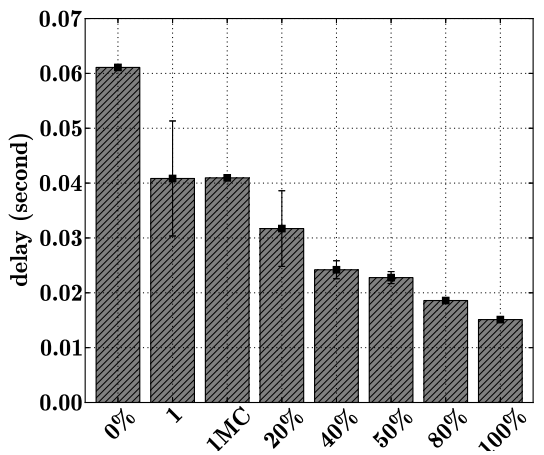

(c) Delay

Fig. 4: Results of experiment with the Abilene topology. (a) \#Interest messages; (b) \#Data messages; (c) Delay

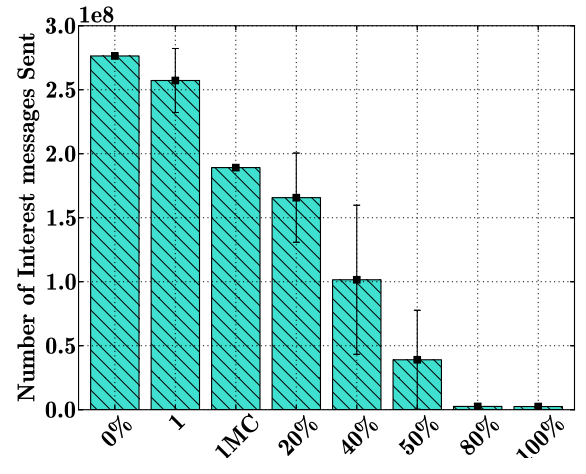

(a) Messages Interest

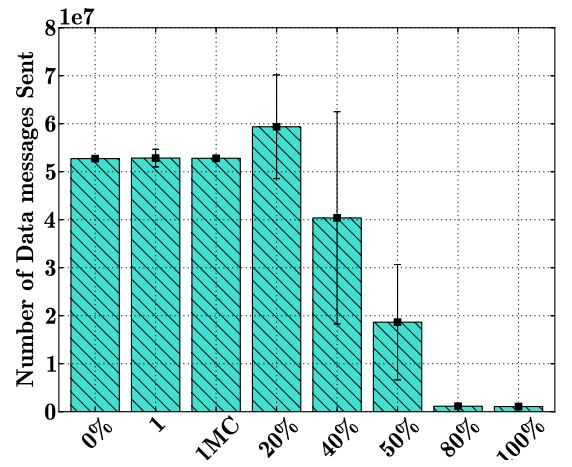

(b) Messages Data

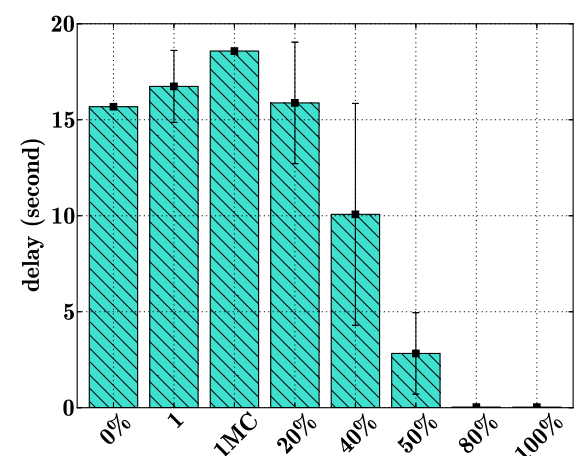

(c) Delay

Fig. 5: Results of experiment with the Geant topology. (a) \#Interest messages; (b) \#Data messages; (c) Delay

Interest and the reception of the corresponding Data message (e.g.: similar to a Round-Trip Time in IP network, and taking into account Interest re-transmissions). For both topologies, the delay decreases with regards to the number of Content Stores. This result was expected because more Content Stores will be close to users, reducing the distance (i.e. the delay) to reach a content. However, the delay decreases drastically up to $50 \%$ of cache nodes and then decrease more slowly. Again, the more Content Stores does not necessarily improve significantly the performances and there is no need having Caching capabilities at each nodes.

\section{Discussion And NeXt Steps}

Our results show that a NDN network can reach high level of performances without having Content Stores at each node. Then, there is a trade-off between the number of Content Stores in the network and the performances. This is a counter-intuitive finding as one could have expected that the more Content Stores will lead to higher performances in the network. From a network provider perspective, this finding is important since reducing the number of Content Stores will help reducing the cost of the entire infrastructure, as memory is the main cost of new hardware equipment. Indeed, with "Green Growth", efficient use of resources allows achieving higher performances than deploying costly infrastructure (e.g.: cache memories) without any preliminary studies or consideration on the resource environment. This result is therefore an important step toward real deployment of the NDN architecture at largescale.

Thus, future deployment of NDN infrastructure may consider a network with only a subset of nodes equipped with cache capabilities, as having Content Stores on each node does not increase the performances of the network.

Remind that we performed our experiments by using two different ISP-like topologies (Abilene and Geant), we noticed that it has an impact on the performances. For Abilene, Scenario with $40 \%$ of cache nodes achieves the highest Cache Hit, while reducing drastically the number of the messages in the network. For Geant, even though Scenario 40\% achieves the highest Cache performances (Cache Hit Ratio), Scenario with 50\% allows reducing drastically the number of messages in the network while keeping a high Cache Hit (Figures 4 and 6). Reducing the number of messages, as well as the Content Stores memory will have a direct impact on the entire infrastructure cost, but also energy consumption etc. Network providers will directly benefit from this result as the entire cost for deploying such novel architecture is reduced (CAPEX), as well as inherent cost for maintaining a network infrastructure (OPEX). 
In our results, we have also observed that the Content Stores placement also impacts the performances of the network. Indeed in Scenarios with a single Content Store ("1" and "1MC"), the performances are higher when Content Store is located on the most-connected node, and it is especially the case on the well-connected Geant topology. As we find out that a reduced set of Content Stores allows achieving highest performances, an open question for future work would be where to locate the Content Stores in a given topology in order to achieve the best performances. Thus, future work may consider new location strategies to deploy Content Stores. Considering the most connected nodes is obviously an option; however, such nodes may receive a lot of traffic from others, consuming their resources and performing a lot of caching operations, which can have opposite effect and limit the overall performances at the end. Besides, users access the Internet mostly with mobile devices. This fact should help designing novel Content Store location strategies for NDN. As there is no need to deploy caching capabilities at each node, caching only in the core network, or closer to the users and mobile equipment (Base Station, etc.) should be considered by telco companies.

Content Popularity is also a concern and it is very important to model it accurately. Indeed, if content popularity is uniform, then Content stores will be frequently changed as there is no consensus about any popular content, making de-facto useless the need for a cache network architecture. Differently, if some content is very popular,caching this content close from users is essential and will save resources as it is currently the case with the use of CDN in IP network. Thus, Content Store location and popularity modeling are still very important question to investigate, while caching strategies or NDN forwarding scheme have already attracted a lot of attention from the research community.

Further step will be to entail our methodology and evaluate the energy saving of a deployed NDN network by reducing the number of Content stores in NDN nodes. Indeed, environment is now at the core of the inter-governmental discussions (COP21 in 2015), and energy saving is at the core of the debate.

\section{CONCLUSION}

In this paper, we have investigated the impact of Content Stores in NDN network. We have performed extensive simulations with NS-3/ndnSim and evaluated the performances of NDN network with different caching capabilities. Our results show that only $50 \%$ of nodes with caching capabilities (i.e.: Content Stores) achieve the best performances in term of Cache Hit, number of messages and delay. Thus, this is not necessary to have all the nodes equipped with Content Stores; as memory is one of the main infrastructure cost for NDN, this result shows that a large-scale deployment can be considered by network providers at reduced costs.

As discussed in previous section, future work should entail the energy reduction with less Content Stores in the network. Content Stores location strategies, as well as content popularity modelling and forwarding scheme are still interesting topics to be investigated.

\section{REFERENCES}

[1] V. Jacobson, D. K. Smetters, J. D. Thornton, M. F. Plass, N. H. Briggs, and R. L. Braynard. Networking named content. ACM CoNEXT 2009.

[2] L. Zhang, A. Afanasyev, J. Burke, V. Jacobson, KC Claffy, P. Crowley, C. Papadopoulos, L. Wang, and B. Zhang. Named data networking. ACM SIGCOMM 2014.

[3] W.K. Chai, D. He, I. Psaras, and G. Pavlou. Cache "less for more" in information-centric networks. Computer Communications, 36(7):758 770, 2013.

[4] C. Bernardini, T. Silverston, and O. Festor. MPC: Popularity-based caching strategy for content centric networks. IEEE ICC 2013.

[5] C. Bernardini, T. Silverston, and O. Festor. Socially-aware caching strategy for content centric networking. In IFIP Networking 2014.

[6] J. Ren, W. Qi, C. Westphal, J. Wang, K. Lu, S. Liu, and S. Wang. Magic: A distributed max-gain in-network caching strategy in informationcentric networks. In IEEE INFOCOM WKSHPS 2014.

[7] M. Rezazad and Y.C. Tay. Ccndns: A strategy for spreading content and decoupling ndn caches. In IFIP Networking 2015.

[8] E.J. Rosensweig and J. Kurose. Breadcrumbs: Efficient, best-effort content location in cache networks. In IEEE INFOCOM 2009.

[9] M. Rezazad and Y. C. Tay. A cache miss equation for partitioning an ndn content store. In ACM AINTEC 2013.

[10] Y. Wang, Z. Li, G. Tyson, S. Uhlig, and G. Xie. Optimal cache allocation for content-centric networking. In IEEE ICNP 2013.

[11] D. Rossi and G. Rossini. On sizing cen content stores by exploiting topological information. In IEEE INFOCOM WKSHPS 2012.

[12] S.K. Fayazbakhsh, Y. Lin, A. Tootoonchian, A. Ghodsi, T. Koponen, B. Maggs, K.C. Ng, V. Sekar, and S. Shenker. Less pain, most of the gain: Incrementally deployable icn. ACM SIGCOMM 2013.

[13] Y. Wang, Z. Li, G. Tyson, S. Uhlig, and G. Xie. Optimal cache allocation for content-centric networking. In IEEE ICNP 2013.

[14] D. Perino and M. Varvello. A reality check for content centric networking. In ACM ICN SIGCOMM WKSHPS 2011.

[15] X. Jiang and J. Bi. ncdn: Cdn enhanced with ndn. In IEEE INFOCOM WKSHPS 2014.

[16] T.Y. Wu, Y.W. Wu, and K.L. Cheng. An efficient ndn-based load adjustment scheme for reduction of energy consumption. In IEE CIT 2014.

[17] Cisco vni. http://www.cisco.com/c/en/us/solutions/service-provider/ visual-networking-index-vni/index.html.

[18] E. Aubry, T. Silverston, and I. Chrisment. SRSC: SDN-based Routing Scheme for CCN. In IEEE NetSoft 2015.

[19] A.K.M. Hoque, S.O. Amin, B. Alyyan, A.and Zhang, L. Zhang, and L. Wang. Nlsr: Named-data link state routing protocol. In $A C M$ SIGCOMM ICN WKSHPS 2013

[20] M. Jakob and O. Edenhofer. Green growth, degrowth, and the commons. Oxford Review of Economic Policy.

[21] NS-3 Consortium. projet ns-3. https://www.nsnam.org/.

[22] C. Bernardini. Stratégies de Cache basées sur la popularité pour Content Centric Networking. PhD thesis, Université de Lorraine, 2015.

[23] D. Rossi and G. Rossini. Caching performance of content centric networks under multi-path routing (and more). Relatório técnico, Telecom ParisTech, 2011.

[24] I. Psaras, W. K. Chai, and G. Pavlou. In-network cache management and resource allocation for information-centric networks. IEEE Transactions on Parallel and Distributed Systems 2014. 